14

\title{
Определение поглощенной в биологическом образце дозы при смешанном гамма-нейтронном облучении
}

\author{
(C) C.С. Арзуманов, ${ }^{1}$ В.В. Сафронов, ${ }^{2}$ А.Н. Стрепетов ${ }^{1}$ \\ ${ }^{1}$ Национальный исследовательский центр „Курчатовский институт“, \\ 123182 Москва, Россия \\ 2 Федеральный научно-исследовательский центр „Кристаллография и фотоника“ РАН, \\ 119333 Москва, Россия \\ e-mail: Arzumanov_SS@nrcki.ru
}

(Поступило в Редакцию 29 ноября 2017 г.)

Описана процедура и представлены результаты определения поглощенных доз при смешанном гамма-нейтронном облучении биологических образцов на горизонтальном канале исследовательского реактора ИР-8 НИЦ „Курчатовский институт“.

DOI: 10.21883/JTF.2018.10.46505.2580

При сравнении эффектов воздействия излучения на биологическую ткань авторы приводят условия облучения и поглощенные в образцах биоткани дозы [1-5]. При этом для корректного сравнения необходимо знать не только тип излучения, но и плотность потока излучения, его спектральный состав, геометрические размеры образца. А при смешанном, например, гамма-нейтронном облучении отдельно определять вклад в поглощенную дозу каждого вида излучения.

В настоящей работе описывается процедура определения поглощенной дозы при смешанном гамма-нейтронном облучении образцов мезенхимальных стволовых клеток (МСК) мышей. Облучение проводилось на горизонтальном экспериментальном канале (ГЭК-1) реактора ИР-8 НИЦ „Курчатовский институт“. Нейтронное и гамма излучения от активной зоны реактора формировались коллиматором из стали и борированного полиэтилена. Диаметр выходного отверстия коллиматора равнялся $14 \mathrm{~mm}$. Образцы МСК в культуральной жидкости в тонкостенном пластиковом флаконе размещались непосредственно на выходе коллиматора. Поглощенные при облучении дозы (раздельно для нейтронного и гамма излучений) вычислялись методом Монте-Карло с использованием программного пакета Geant4 [6-8] версии 10.1.2. Электромагнитные взаимодействия моделировались с помощью входящего в этот пакет набора физических процессов G4EMLOW, применимого для диапазона энергий фотонов от $10 \mathrm{eV}$ до $100 \mathrm{GeV}$. Для моделирования взаимодействий нейтронов применялся список физических процессов QGSP_BIC_HP, также входящий в состав Geant4, позволяющий моделировать взаимодействие с веществом нейтронов с энергиями вплоть до нескольких десятков $\mathrm{MeV}$. В расчетах учитывались форма и состав образца, спектры и плотности нейтронного и гамма потоков.

Плотность нейтронного потока в выведенном пучке была измерена методом водяного куба [9] и равнялась $(7.2 \pm 0.6) \cdot 10^{7} \mathrm{~cm}^{-2} \mathrm{~s}^{-1}$ при мощности реак- тора 6.2 MW. Точность измерения определяется паспортной точностью активности нейтронного источника, используемого при калибровочных измерениях. Постановка в пучок кадмиевого фильтра толщиной $1 \mathrm{~mm}$ показала, что доля нейтронов, имеющих энергию больше $0.5 \mathrm{eV}$, составляет $61 \%$. Облучение образцов проводилось с использованием кадмиевого фильтра, соответственно плотность нейтронного потока была равна $(4.4 \pm 0.4) \cdot 10^{7} \mathrm{~cm}^{-2} \mathrm{~s}^{-1}$. В расчетах принималось, что энергетический спектр этих нейтронов - это спектр Ферми, для которого зависимость нейтронного потока $N(E)$ от энергии нейтронов $E$ имеет вид $N(E) \propto 1 / E[10,11]$. Это выражение справедливо при замедлении нейтронов в гомогенной слабо поглощающей среде на легких ядрах в реакторах на тепловых нейтронах. Реактор ИР-8 - это реактор водо-водяного типа, замедлителем является вода и бериллий отражателя, в котором начинается ГЭК-1. Верхняя граница нейтронного спектра по энергии $\sim 10 \mathrm{MeV}$ (граница энергетического спектра нейтронов при делении $\left.{ }^{235} \mathrm{U}\right)$.

Мощность реактора в различных сериях облучения образцов менялась от 4.8 до $6.5 \mathrm{MW}$. Полагалось, что нейтронный и гамма потоки в этом интервале пропорциональны мощности реактора.

Образцы клеток в культуральной жидкости в течение нескольких минут оседали и собирались в клубок диаметром примерно $4 \mathrm{~mm}$ вблизи дна флакона. Поэтому в расчетах поглощенной дозы использовался фантом в виде цилиндра диаметром и длиной $4 \mathrm{~mm}$. Эта форма геометрически близка к форме используемого флакона. В реальности столб жидкости во флаконе был примерно на $10 \mathrm{~mm}$ выше интересующей нас области, однако расчеты показали, что на величину поглощенной дозы он практически не влияет.

На рис. 1 представлена расчетная зависимость мощности поглощенной в фантоме дозы $D_{n}$ при облучении нейтронами в энергетическом диапазоне $0.5-1 \cdot 10^{7} \mathrm{eV}$ от расстояния $d$ по оси от основания цилиндра, через 


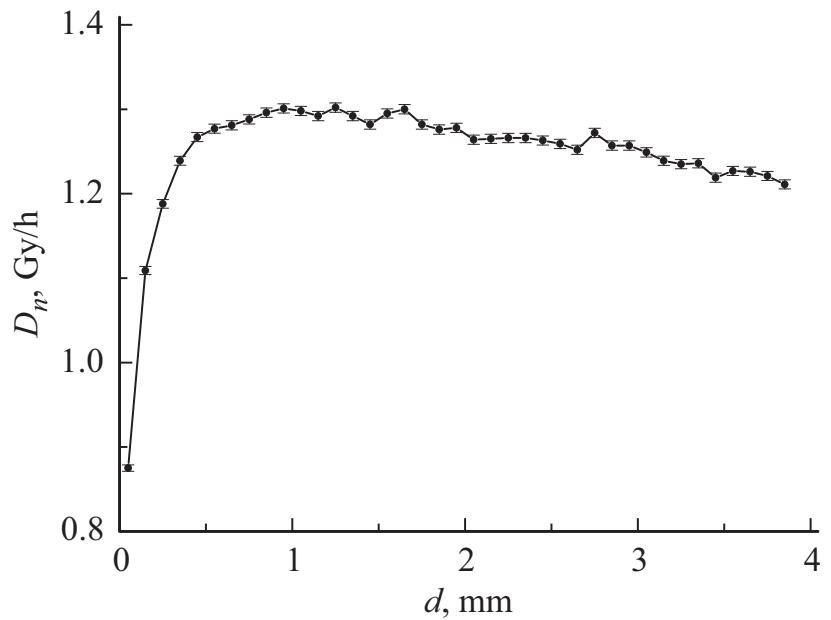

Рис. 1. Расчетная зависимость мощности поглощенной дозы от расстояния $d$ при облучении образца нейтронами.

которое происходит облучение. Здесь и во всех расчетах ниже направление пучка совпадает с осью цилиндрического фантома, и расчеты выполнены для фантома из воды.

Из рисунка видно, что мощность поглощенной дозы максимальна при $d \approx 0.9 \mathrm{~mm}$ и затем медленно падает с ростом $d$, уменьшаясь на 5-7\% при $d=4 \mathrm{~mm}$. Среднее по объему значение мощности поглощенной дозы составляет $1.24 \pm 0.02 \mathrm{~Gy} / \mathrm{hr}$. С учетом того что изначально плотность нейтронного потока была определена с точностью примерно $8 \%$, за мощность поглощенной дозы при нейтронном облучении принята величина $1.24 \pm 0.10 \mathrm{~Gy} / \mathrm{hr}$ при мощности ядерного реактора $6.2 \mathrm{MW}$.

В расчете поглощенной дозы при облучении образцов гамма-квантами основная трудность заключалась в определении плотности потока и спектрального состава пучка гамма-квантов работающего реактора. При решении этой задачи был использован подход, основанный на дозиметрических измерениях.

Прежде всего, был определен диапазон энергий гамма-квантов. На пути от активной зоны реактора до входа в коллиматор находятся примерно $10 \mathrm{~cm}$ воды, $10 \mathrm{~cm}$ бериллия, $0.5 \mathrm{~cm}$ циркония и $3 \mathrm{~m}$ воздушного промежутка. Поэтому в коллиматор попадают гамма-кванты с энергией не меньше $0.1 \mathrm{MeV}$. Двигаясь по коллиматору, они испытывают комптоновское рассеяние на стенках, при этом в пучке остаются только гамма-кванты, рассеянные на малые углы, и соответственно мало меняющие свою энергию. Выбор верхней границы спектра основан на результатах расчетных работ [12-14], которые показывают практическое отсутствие гамма-квантов с энергиями, большими 3-4 MeV. Исходя из сказанного, дальнейшие расчеты были выполнены для диапазона энергий гамма-квантов $0.1-4.0 \mathrm{MeV}$.

Далее была измерена зависимость $H(x)$ мощности амбиентной дозы (МАД), создаваемой пучком гамма-квантов, проходящих сквозь свинцовые экраны различной толщины $x$ (рис. 2). В измерениях использовался дозиметр Radiagem 2000 фирмы Canberra с блоком детектирования STTC, который может работать в больших гамма потоках. Дозиметр фиксировался на пучке вблизи выхода коллиматора в положении наибольшего счета. Затем снимались его показания при постановке на выходе коллиматора свинцовых экранов различной толщины. Мощность реактора при измерениях была равна 6.5 MW. Сплошная кривая на рис. 2 - это аппроксимация экспериментальных точек экспонентой. Из параметров аппроксимации следует, что пучок в целом с хорошей точностью ведет себя как пучок с узким энергетическим диапазоном с энергией гамма-квантов $E_{\gamma} \sim 2.1 \mathrm{MeV}$.

Следующим шагом весь интервал энергий гамма-квантов был поделен на подынтервалы разной ширины по числу используемых свинцовых экранов. Ширины подынтервалов определялись видом зависимости линейного коэффициента ослабления потока гамма-квантов

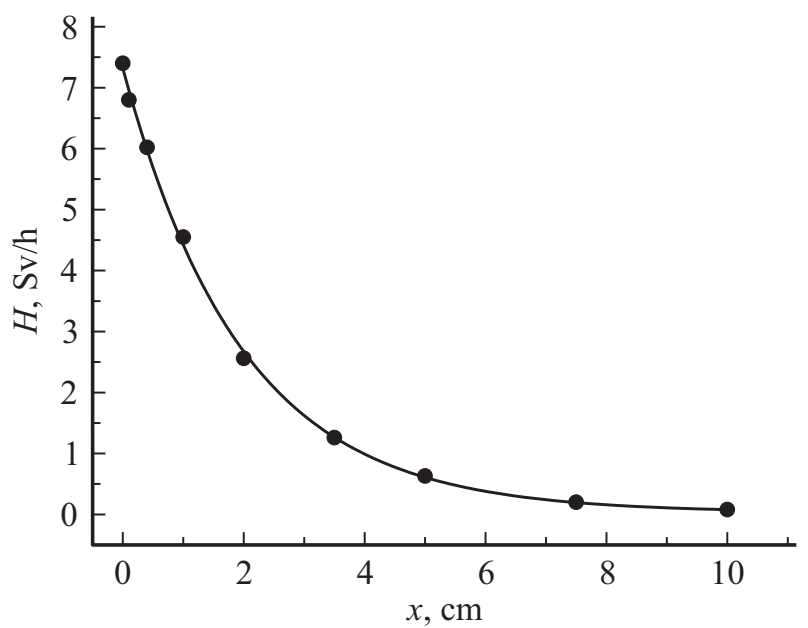

Рис. 2. Измеренная зависимость МАД создаваемой исследуемым пучком гамма-квантов, проходящим сквозь свинцовые экраны, от толщины экрана.

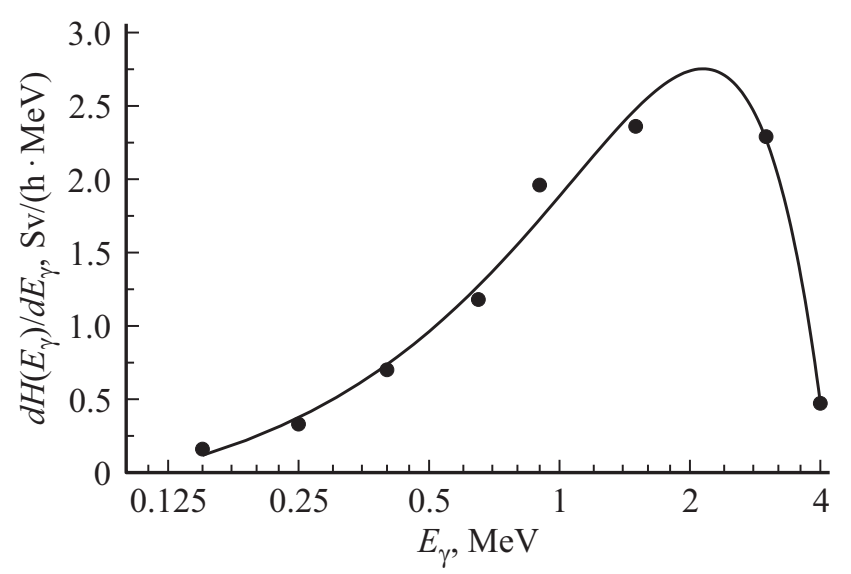

Рис. 3. Дифференциальный спектр МАД на выходе коллиматора. 


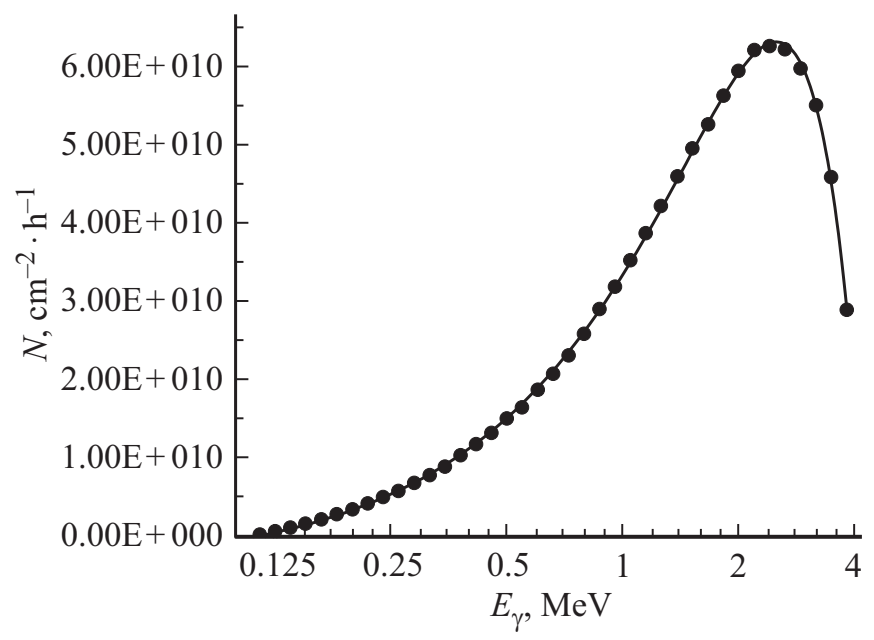

Pис. 4. Восстановленная зависимость плотности потока гамма-квантов от энергии в месте облучения образцов.

$\mu\left(E_{\gamma}\right)$ от $E_{\gamma}$ и в каждом из них $\mu\left(E_{\gamma}\right)$ считалась постоянной. Тогда для каждого подынтервала закон ослабления МАД свинцовым экраном будет иметь вид $H=H_{0} \exp \left[-\mu\left(E_{\gamma}\right) x\right]$, где $H_{0}-$ МАД падающего пучка. Используя это соотношение, а также данные по ослаблению пучка гамма-квантов свинцовыми экранами, можно для каждого подынтервала восстановить значения МАД. На рис. 3 эти значения МАД, пересчитанные на единичный интервал энергии, обозначены точками. Сплошная кривая - аппроксимация полученных значений гладкой кривой.

Далее в диапазоне энергий гамма-квантов 0.1-4.0 MeV были выбраны 39 значений $E_{\gamma}$ с логарифмическим распределением внутри диапазона. Для каждого значения $E_{\gamma}$ рассчитывалась соответствующая МАД. Затем в соответствии с определением МАД выполнялся расчет плотности потока гамма-квантов $\mathrm{N}$ с энергией $E_{\gamma}$, необходимого для получения мощности поглощенной дозы, равной МАД для данной энергии. Расчеты выполнялись для цилиндрического (диаметром и длиной $30 \mathrm{~cm}$ ) водного фантома, близкого по свойствам шаровому фантому МКРЕ, для точки, расположенной на оси цилиндра на расстоянии $10 \mathrm{~mm}$ от основания [15]. Поток гамма-квантов направлялся также по оси цилиндра. Полученный таким образом энергетический спектр плотности потока гамма-квантов представлен на рис. 4. Максимум спектра приходится на диапазон энергий $2.0-2.5 \mathrm{MeV}$ и, по-видимому, в значительной степени такая форма спектра обеспечивается вкладом гамма-квантов с энергией $E_{\gamma}=2.23 \mathrm{MeV}$ из реакции поглощения нейтронов на водороде воды.

С целью проверки восстановленного спектра был выполнен расчет мощности поглощенной дозы для 30-сантиметрового водяного фантома на расстоянии $10 \mathrm{~mm}$ от основания по оси. Полученная величина $7.5 \mathrm{~Gy} / \mathrm{hr}$ хорошо согласуется с экспериментально изме- ренной мощностью амбиентной дозы $7.4 \mathrm{~Sv} / \mathrm{hr}$ (показания дозиметра без свинцового экрана на пути пучка).

С этим спектром был выполнен расчет мощности поглощенной дозы $D_{\gamma}$ в водном фантоме диаметром и длиной $4 \mathrm{~mm}$, принятом за аналог облучаемых образцов, а также в водном фантоме диаметром и длиной $30 \mathrm{~cm}$. Как и в ранее выполненных расчетах, использовался пакет Geant4. Результаты расчета приведены на рис. 5. Как видно из рисунка, мощность поглощенной дозы для 30-сантиметрового фантома заметно превышает аналогичную величину для 4-миллиметрового фантома, что связано с увеличением доли перерассеянных гамма-квантов, дающих вклад в поглощенную дозу. Также из рисунка видно, что наблюдается заметное увеличение поглощенной дозы при удалении от основания. Это означает, что в процессе облучения необходимо обеспечить перемешивание раствора, содержащего образцы клеток.

Среднее по объему значение мощности поглощенной дозы при облучении гамма-квантами для 4-миллиметрового фантома равно $2.47 \mathrm{~Gy} / \mathrm{hr}$. Учитывая, что дозиметрические измерения не обеспечивают точность лучше $20 \%$, окончательному результату мощности поглощенной дозы приписывается точность в 20\%. Статистическая погрешность всех выполненных расчетов на порядок ниже.

Окончательно в пересчете на $1 \mathrm{MW}$ мощности реактора имеем

$$
\bar{D}_{n}=0.20 \pm 0.02 \mathrm{~Gy} / \mathrm{hr}, \quad \bar{D}_{\gamma}=0.38 \pm 0.08 \mathrm{~Gy} / \mathrm{hr} .
$$

Аналогичный цикл измерений и расчетов был выполнен с использованием дозиметра-радиометра МКС-АТ1117М с блоком детектирования БДКГ-01. Значение средней мощности поглощенной дозы в пересчете на $1 \mathrm{MW}$ мощности реактора составило $\bar{D}_{\gamma}=$ $=0.45 \pm 0.09 \mathrm{~Gy} / \mathrm{hr}$. Таким образом, значения $\bar{D}_{\gamma}$, полученные с использованием двух различных дозимет-

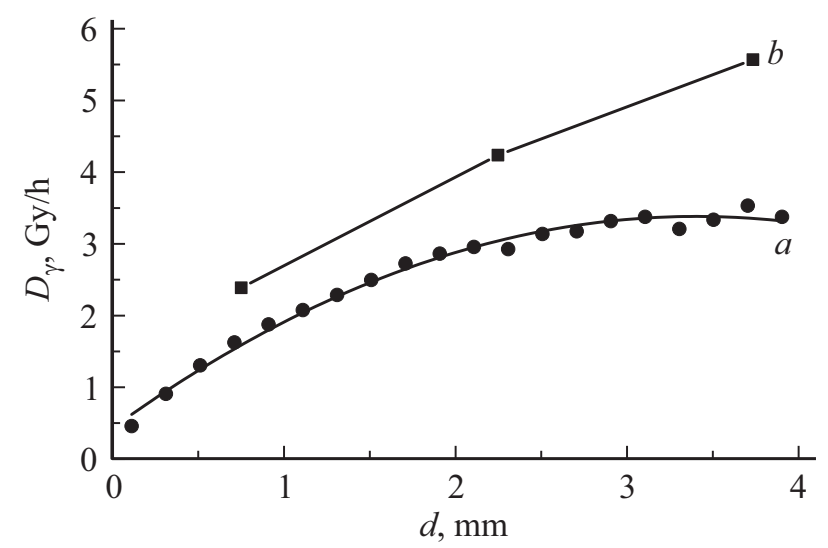

Рис. 5. Расчетная зависимость мощности поглощенной дозы при облучении образца гамма-пучком от расстояния $d$ вдоль оси цилиндра для 4-миллиметрового фантома $(a)$ и начальная часть такой же зависимости для 30-сантиметрового фантома $(b)$. 
рических приборов, в пределах заявленной точности непротиворечивы.

Знания величин $\bar{D}_{n}, \bar{D}_{\gamma}$ и мощности реактора позволяют определить время облучения образцов для задаваемой поглощенной дозы.

Работа выполнена при частичной поддержке гранта РФФИ 15-29-01234-офи_м.

\section{Список литературы}

[1] Ульяненко С.Е., Лычагин А.А., Соколов В.А. и др. // Медицинская физика. 2006. № 4. С. 10-17.

[2] Матчук О.Н., Замулаева И.А., Селиванова Е.И. и др. // Радиационная биология. Радиоэкология. 2012. Т. 52. № 3. C. 261-267.

[3] Москалева Е.Ю., Жорова Е.С., Семочкина Ю.П. и др. // Цитология. 2017. Т. 59. № 4. С. 271-278.

[4] Посыпанова Г.А., Москалева Е.Ю., Родина А.В. и др. // Радиационная биология. Радиоэкология. 2016. Т. 56. № 1. C. 35-43. DOI: $10.7868 / 50869803116010124$

[5] Ульяненко С.Е., Корякин С.Н., Исаева Е.В. и др. // Радиационная биология. Радиоэкология. 2016. Т. 56. № 6. C. 598-605. DOI: $10.7868 / 5086980311606014 \mathrm{X}$

[6] Электронный ресурс. Режим доступа: http:/geant4.cern.ch

[7] Agostinelli S., Alligon J., Forti A. et al. // Nucl. Instr. Meth. Phys. Res. 2003. Vol. A506. N 3. P. 250-303. DOI: $10.1016 / 50168-9002(03) 01368-8$

[8] Allison J., Walkden A., Amako K. et al. // IEEE Trans. Nucl. Sci. 2006. Vol. 53. N 1. P. 270-278. DOI: $10.1109 / \mathrm{TNS} .2006 .869826$

[9] Арзуманов С.С., Морозов В.И., Панин Ю.Н., Стрепетов А.Н. Ноу-хау НИЦ „Курчатовский институт“. Рег. № $143 / 2013$ от 28.03 .2013 .

[10] Баранник А.А. Лекции по курсу „Теория переноса нейтронов“. М.: НИЯУ МИФИ, 2012. 164 с.

[11] Гуревич И.И., Протасов В.П. Нейтронная физика. М.: Энергоатомиздат, 1997. $416 \mathrm{c}$.

[12] Гарусов Е.А., Грачев С.Д., Петров Ю.В. Препринт ЛИЯФ № 1473. Ленинград. 1989. 25 c.

[13] Брискман Б.А., Бондарев В.Д., Захаров Л.Н. // Атомная энергия. 1974. Т. 37. № 3. С. 208-211. [Briskman B.A., Bondarev V.D., Zakharov L.N. // Atomic Energy. 1974. Vol. 37. N 3. P. 920-924.].

[14] Комелев А.С., Хоруджий В.Л. // ВАНТ. Серия: Физика ядерных реакторов. 2013. № 3. С. 65-82.

[15] Комочков М.М. Дозиметрия ионизирующих излучений. Дубна: Международный университет природы, общества и человека, 2006. $76 \mathrm{c}$. 